\title{
Research on the Reciprocating Motion of the Man-Machine Indirect Piston and the Efficiency of Internal Friction
}

\author{
Yi-Fan ZHAO ${ }^{\mathrm{a}}$, Hua LI ${ }^{\mathrm{b}}$, Hung-Ming CHANG ${ }^{\mathrm{a}}$, Chih-Chung LIN ${ }^{\mathrm{a}}$, Shinn-Dar WU, ${ }^{\mathrm{c}}$ \\ and Chia-Ming $\mathrm{CHANG}^{\mathrm{d}}$ \\ ${ }^{a}$ Fufeng Material Technology Co., Ltd, Taiwan, China \\ b Yunnan Art Institute, Yunnan Kunming, Kunming, China \\ c School of Chemistry and Materials Engineering, Huizhou University, Guangdong, \\ China \\ ${ }^{\mathrm{d}}$ CNT Educational Institution, Taiwan, China
}

\begin{abstract}
In the process of technological progress and development, many industries are moving towards artificial intelligence (AI) technology, which is a construction and combination of multi-thinking technology. But in terms of structure, material development and diversification, the source of ideas is still inseparable from the natural world. Therefore, when faced with solving problems, many scientists not only conduct in-depth research on changes in cells, tissues and expansion, to solve the multi-dimensional movement of current technology, but also non-destructively explore the thermal effects of friction. Self-generated lubrication or external additives are used to solve the demand, so that the reciprocating movement of the piston can be more effective, and each cycle can be achieved. According to research and discussion, in each cycle, heat engine and cooling, speed movement frequency and lubrication method are different, which not only makes the piston have different effects, but also the reaction and sensitivity will greatly change, which makes it necessary to stop resting. Therefore, this research is primarily aimed at exploring the reciprocating motion of the human-machine "Indirect piston" and the efficiency of internal friction. The purpose is to have a more in-depth study of the piston theory, so as to have a deeper foundation for the movement of derived multi-dimensional angles in the future. In the future, there will be better development in injection, piston mechanism, and lubrication.
\end{abstract}

Keywords. Injection, piston movement, lubricant, friction.

\section{Introduction}

The advancement and development of science and technology has reached the Ai field, requiring more scientific and technological ideas from nature and human behavior. Many creations and inventions were born as a result, especially the basic principles of movement, which are mainly derived from the movement of pistons to produce the future.

${ }^{1}$ Corresponding Author, Shinn-Dar WU, School of Chemistry and Materials Engineering, Huizhou University, Guangdong, China; E-mail: 13478511396@139.com. 
In kinematics, there are about three most common piston motions [1-2]: the first, the fore body pose is a more common motion posture, which refers to the movement of the piston from top to bottom, just like the stent expands outward, and the piston sucks the oil well. The opposite situation can also be called the superstructure of the subject, while Western countries are called the fore body pose (Missionary Position); The second type of cupping or upper position, such as the engine piston, means that the main force of the posture comes from the flexion and extension of the hip joint. The stability of the core is very important. If it is not stable enough, it may cause excessive torso shaking back and forth; The third back position, back position, injection machine principle. This posture usually has two main points. One is the range of motion of the hip joint. If the hip joint is too tight, problems and risks such as waist arching may occur. Piston movement has more than $95 \%$ interaction in the world, and the automation of the robot between the two bodies is introduced, which is mainly responsible for the friction and lubrication between the pistons. In order to avoid obstacle signals in sequence control, a programmable solution is proposed, and its working principle and structure are analyzed. The multipiston motion sequence queuing method and standard design program based on the stepping method are studied, and the design is more convenient [3-4]. A water-soluble lubricant and a preparation method thereof. The water-soluble lubricant comprises 5$20 \%$ glycerin, $5-20 \%$ propylene glycol, $0.1-1.0 \%$ polyacrylate and $0.1-1.0 \%$ by mass percentage. The hydroxypropyl methyl cellulose is used as a thickener, $0.1-1.0 \% \mathrm{pH}$ adjuster, $0.1-0.5 \%$ preservative, and the rest is water. The lubricant has similar viscosity and acidity to the natural lubricating liquid, the mucous membrane is non-toxic, nonirritating, and easy to clean [5].

The common problems in friction work, combined with the friction work problems encountered in the author's teaching, and analyzed according to the latest national new curriculum standards, the characteristics of the work completed by a single friction and the characteristics of the work completed by the friction. Researched and explored a pair of friction force, the work done by static friction force during movement, the purpose is to promote the development and perfection of physical friction teaching [6]. The influence of material friction coefficient and wear rate with temperature, the interaction of each component in the friction material on the friction and wear performance, and the friction and wear characteristics and mechanisms of the friction material are discussed [7]. The Japanese dean Tatsumi Takuro and Kyoko Mitsui jointly created a combination of methods and techniques, including the joint research of the piston movement method, and through interviews and sharing of amateur experience, answering the questions faced by many people, sex refers to the emotional and intentional sexual intimacy of higher intelligent animals (such as humans) with each other. It is a beautiful sexual behavior with love and happiness. It is different from pure physical intercourse and even more different from parents. Love, the love of friends. Man-Machine is a great and important driving force for the continuation and development of society. Man-Machine is a matter of entertainment for men and women. Sex and love are a perfect unity. It is not just an act of sexual intercourse. Man-Machine is different from the vulgar and despicable physical possession. The real purpose of sex is to express and pursue the happy state of inner love. Through research, sex experts found that sex is a natural tranquilizer and analgesic, which has the function of improving the immune system and prolonging life [8-9]. When the driving part of a mechanism moves at a constant speed (rotating or swinging), while the driven part is sometimes static and sometimes moving, this kind of mechanism is called an intermittent motion mechanism [10]. 
Therefore, this research will explore the relevance of piston motion, the influence of lubrication and the thermal effect. The purpose of this research is to use the best recirculation technique to activate the traditional piston principle and provide a new piston solution under the diversification for reference.

\section{Theoretical Discussion}

This study uses the traditional piston to explore the principle of "indirect piston" action, as shown in figure 1, which is used for the speed and acceleration reaction mechanism and forms a special procedure. This study preliminarily explores the capillary phenomenon and lubrication points on the inner wall of the piston. In order to complete the research of enterprise experimental design requirements.

Based on the needs of the enterprise, combined with academic research, the phenomenon and analysis of the indirect piston have been initially completed. This research will implement the integration of industry and academics to achieve the basis for practical application and future material improvement.

The reason for the preliminary development of this research is to propose technical solutions and product designs based on the state of the cycle mechanism, for the technical solutions brought by the enterprise during development. In the future, based on the research foundation of this thesis, it will be extended to explore the friction technology, lubrication technology, air tightness, temperature and speed.

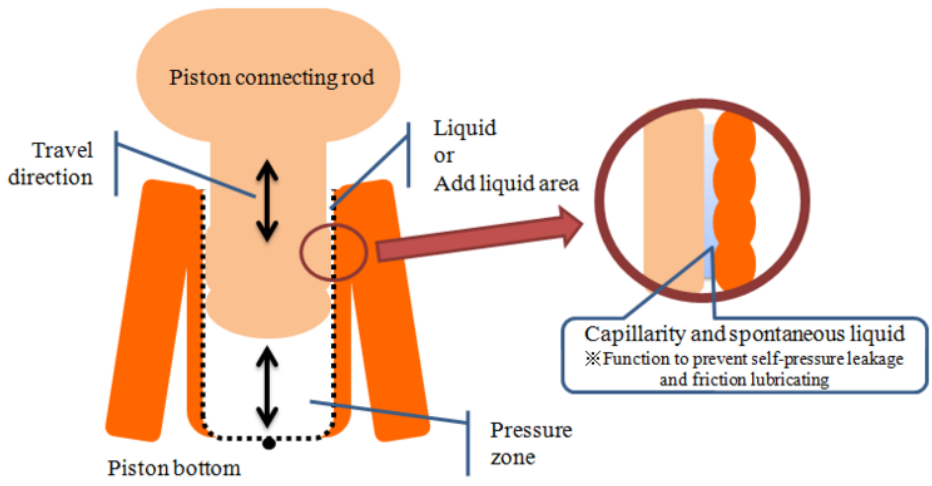

Figure 1. Schematic diagram of new piston exploration.

\section{Comprehensive Analysis}

According to the experimental data shown in figure 2, when the indirect piston is about 7-9 minutes, the material is in a close state due to expansion. At an instantaneous speed, the friction within the heat engine generates a temperature increase. After the lubricating fluid is kept for a few minutes, the concentration will increase or disappear. This is because the piston body changes in the speed difference, and the speed will drop in about 10 minutes.

At about 11 minutes, the high-speed operation resumed when the temperature and lubrication were in equilibrium, and the speed slowed down again at about 19 minutes. 
At this time, 1-2ml of lubricating fluid should be added to the number (2) in figure 2 . At about the 28th minute, number (3) in figure 2 has the maximum and stable efficiency, which is also the maximum work of the piston. Meet the company's product technology development, indirect piston response and procedures.

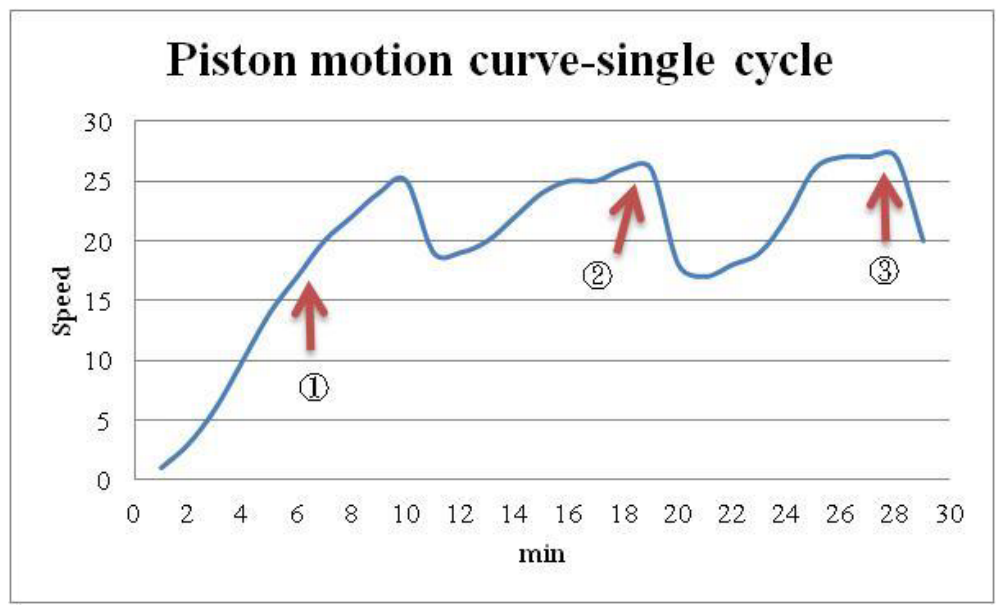

Figure 2. Trend analysis of piston single cycle experiment.

After being operated by a single machine, the multi-cycle experiment is shown in figure 3. In the numbers (1), (2) and (3), the second cycle (5), (6) and (7) have the same reaction state, and the number (4) is the preparation for replacement after the first cycle, and preparation for the next cycle.

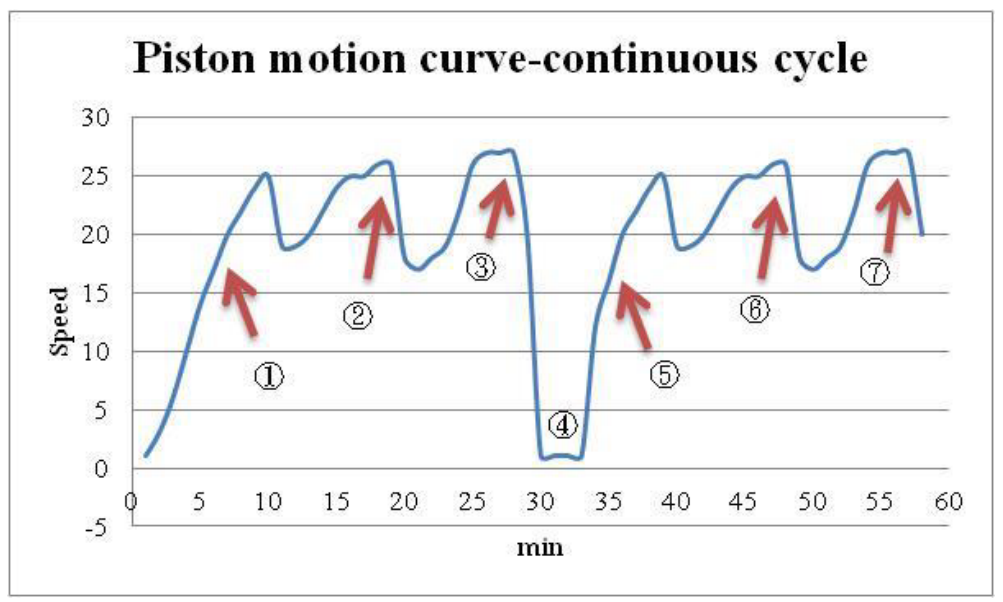

Figure 3. Analysis and discussion of piston multi-circulation experiment.

In this preliminary research process and cyclical experiment exploration, we have a better understanding of friction, temperature, and the relationship between materials at extreme speeds. Before the design of new materials, obtaining this response curve will help in the best conditions required for the modification of materials such as silica gel and EVA in the future. Some people think that the best time is evening. The so-called 
night generally refers to around 22 o'clock. For a considerable number of people, this is the time to fall asleep. People who hold this view believe that activities require a lot of physical effort, and they can fall asleep immediately after the event, so that both parties can get adequate rest and maintain sufficient energy the next day. Some people think that from a physiological point of view, the adrenal glands in the human body in the early morning The concentration of hormones is the highest; some people argue that it is best to sleep for a few hours first and then have sex after waking up. Those who hold this view believe that the pace of life of modern people is relatively fast. After 8 hours of study and work a day, most of them are relatively tired, but at night they want to enjoy the relaxed nightlife and also want to make necessary social interactions. Sleep late at night. Under this situation, the physical strength is low and the spirit is slack. Therefore, the man-machine interface has developed many components from this observation, and the process and machinery have a good man-machine interface to develop many replacements.

\section{Conclusion}

This study has conducted a preliminary study on the piston and the inner wall, and the temperature and lubrication generated during the friction between the two. This study learned from the "indirect piston characteristics" that the immediate replenishment of the internal lubricating fluid is very important when the expansion and velocity change, the capillary phenomenon of the inner wall and its liquid retention feature can add air-tight function, which enables the piston to complete a single cycle smoothly at high speed.

The importance of the human-machine interface is understood from research. The human-machine interface refers to the area where humans and machines contact or influence each other in information exchange and function, or the human-machine interface, information exchange, functional contact or mutual influence, refers to the hard contact and soft touch of human and machine, this joint surface not only includes the direct contact of point, line and surface, but also includes the function space of remote information transmission and control. The human-machine interface is the central link in the human-machine system. It is mainly researched and proposed by the safety ergonomics, a branch of safety engineering, and the basis for the solution. Safety engineering equipment engineering, safety management engineering and safety systems Engineering to study specific solutions, measures, safety ergonomics. It realizes the conversion between the internal form of information and the form acceptable to humans. It is widely used in industry and commerce. It is simply divided into two types: "Input" and "Output". To operate machinery or equipment, such as handles, switches, doors, instructions (commands), or maintenance, etc., and output refers to notifications issued by machinery or equipment, such as faults, warnings, operating instructions, etc., A good man-machine interface will help users operate the machine more simply, more accurately and quickly, and also enable the machine to maximize its performance and extend its service life.

In the future, the friction of the inner wall will be used for microscopic analysis of temperature and lubrication, and the improvement and protection of materials will be discussed in more depth. In the next stage, this thesis will focus on lubricating fluid and material modification as the main direction, and also complete the project technology required by the enterprise. In the future, we will cooperate with human structure, education, relations, psychiatry, linguistics and other cross-fields. 


\section{Acknowledgment}

Special thanks for teamwork, supported by the Professorial and Doctoral Scientific Research Foundation of Huizhou University (2020JB034), and Huizhou Zhongkai HighTech Zone ZongHua Battery Science and Technology Research Institute, and members of the team. Thanks to Chinese culture for providing experimental fields and data verification, so that the core piston motion and mechanism of this article can be discussed in depth.

\section{References}

[1] Piston Movement 2017 https://www.163.com/dy/article/CE59DUTD0528CR7.html.

[2] Wu M-Y 2019 https://www.commonhealth.com.tw/article/article.action?nid=7912.

[3] Ma G-Q, Tian Y-Q, Ma X-H and Guo Y 2018 Study on the design method of total pneumatic sequential control circuit of multi-cylinder based on step forward method Journal of Chinese Corrosion Engineering 32 (2) $17-22$.

[4] Bhat U N and Rao S S 1987 Statistical analysis of queueing systems Queueing Systems 1 217-247.

[5] Cai H, Luo Q, Jiao X and Liu Y 2012 A Water-Soluble Lubricant and Its Preparation Method CN102512338A.

[6] Kelemenová T, Dovica M, Božek P, Koláriková I, Benedik O, Virgala I, Prada E, Miková L, Kot T, Kelemen M 2020 Specific problems in measurement of coefficient of friction using variable incidence tribometer Symmetry 12 (8) 1235.

[7] Su D, Li D, Wang L, et al. 2000 Friction and wear behavior of two semi-metallic friction materials Tribology 20 (5) 383-385.

[8] Zhao B, Dai X, Zhang Z and Xie Y 2016 A new numerical method for piston dynamics and lubrication analysis Tribology International 94 395-408.

[9] Tatsumi T and Kyoko M 2016 Exercise Instruction Manual (Japan: Morning Star Publishing).

[10] Chen W and Chen G 2020 Intermittent Motion Mechanism, General Principles of Mechanical Principles (Introduction), (Hongdian Culture) ISBN: 9789869599269 Unit 15: Intermittent Motion Mechanism. 